\title{
The role of oxygen-increased respirator in humans ascending to high altitude
}

\author{
Guanghao Shen ${ }^{1,2+}$, Kangning Xie ${ }^{2 \dagger}$, Yili Yan², Da Jing ${ }^{2}$, Chi Tang ${ }^{2}$, Xiaoming Wü ${ }^{2}$ Juan Liư ${ }^{2}$, Tao Sun ${ }^{2}$, \\ Jianbao Zhang ${ }^{1 *}$ and Erping Luo ${ }^{2^{*}}$
}

\footnotetext{
* Correspondence: zhangjb@mail. xjtu.edu.cn; luoerping@fmmu.edu.cn 'Equal contributors ${ }^{1}$ Key Laboratory of Biomedical Information Engineering of Ministry of Education, School of Life Science and Technology, Xi'an Jiaotong University, Xi'an, Shaanxi 710049, P. R China

${ }^{2}$ School of Biomedical Engineering, Fourth Military Medical University, Xi'an, Shaanxi 710032, P. R China
}

\begin{abstract}
Background: Acute mountain sickness (AMS) is common for people who live in low altitude areas ascending to the high altitude. Many instruments have been developed to treat mild cases of AMS. However, long-lasting and portable antihypoxia equipment for individual is not yet available.
\end{abstract}

Methods: Oxygen-increased respirator (OIR) has been designed to reduce the risk of acute mountain sickness in acute exposure to low air pressure. It can increase the density of oxygen by increasing total atmospheric pressure in a mask. Male subjects were screened, and eighty-eight were qualified to perform the experiments. The subjects were divided into 5 groups and were involved in some of the tests at 4 different altitudes (Group 1, 2: 3700 m; Group 3,4,5: 4000 m, 4700 m, 5380 m) with and without OIR. These tests include heart rate, saturation of peripheral oxygen $\left(\mathrm{SpO}_{2}\right)$, malondialdehyde (MDA), superoxide dismutase (SOD), blood lactate (BLA) and PWC (physical work capacity) -170.

Results: The results showed that higher $\mathrm{SpO}_{2}$, lower heart rate (except during exercise) and better recovery of heart rate were observed from all the subjects 'with OIR' compared with 'without OIR' $(P<0.05)$. Moreover, compared with 'without OIR', subjects 'with OIR' in Group 1 had lower concentrations of MDA and BLA, and a higher concentration of SOD ( $P<0.05)$, while subjects 'with OIR' in Group 2 showed better physical capacity (measured by the PWC-170) $(P<0.05)$. The additional experiment conducted in a hypobaric chamber (simulating 4,000 m) showed that the partial pressure of oxygen in blood and arterial oxygen saturation were higher 'with OIR' than 'without OIR' $(P<0.05)$.

Conclusions: We suggested that OIR may play a useful role in protecting people ascending to high altitude before acclimatization.

Keywords: Oxygen-increased respirator, Heart rate, Free radical, Acute mountain sickness

\section{Background}

Plateaus, known variously as tablelands, or high altitude, are areas elevated thousands of meters above sea level. With the recent opening of Qinghai-Tibet railway in China, more and more people who live in low altitude areas enter Qinghai-Tibetan high plateau for work, science investigation or tour. Many of them experience acute mountain sickness (AMS), suffering from hypoxia due to the low partial pressure of oxygen at increasing altitudes. The most common symptoms of AMS, including headache, poor

(C) 2012 Shen et al.; licensee BioMed Central Ltd. This is an Open Access article distributed under the terms of the Creative Commons Attribution License (http://creativecommons.org/licenses/by/2.0), which permits unrestricted use, distribution, and reproduction in any medium, provided the original work is properly cited. 
appetite, nausea, fatigue, dizziness and insomnia, usually appear within the first three days of arrival at high altitude [1]. Moreover, people from low altitudes may experience a decrease in exercise performance when ascending to high altitude. The higher the altitude is, the more critical the symptoms may become. This problem may seriously influence the physical and mental state and work efficacy for travelers and workers.

To reduce the risk of AMS, many instruments have been developed, including bottled oxygen [2], portable hyperbaric chamber [3-5] and oxygen enrichment room [6]. The supplemental oxygen provided by these instruments raises the oxygen concentration and reduces the equivalent altitude [7,8], which can treat mild cases of AMS. However, long-lasting and portable anti-hypoxia equipment for individual is not yet available.

In this study, we performed a prospective, self-controlled study to determine the effects of oxygen-increased respirator (OIR, custom-made instrument) on changes in (1) heart rate and saturation of peripheral oxygen $\left(\mathrm{SpO}_{2}\right)$ [9] in four different altitudes; (2) malondialdehyde (MDA), superoxide dismutase (SOD), blood lactate (BLA) [10] at 3,700 m; and (3) PWC (physical work capacity)-170 test [11] at 3,700 m; (4) arterial blood gas analysis at simulated altitude of $4000 \mathrm{~m}$. During each session, subjects performed the experiment with or without OIR.

\section{Materials and methods}

\section{Subjects}

The subjects, coming from a road construction company, were going to high altitude to build roads. After signing informed consent forms, subjects completed a structured questionnaire covering anthropometric variables, lifestyle questions, and medical history. Meanwhile, our research faculties followed them to the high altitude from Xi'an city $(430 \mathrm{~m})$ by vehicles in five days. The field working bases with hotel facilities were distributed into the four different altitudes $(3700 \mathrm{~m}, 4000 \mathrm{~m}, 4700 \mathrm{~m}$, and $5380 \mathrm{~m})$. Accordingly, our research faculties were organized into four teams to carry out the experiment in specific altitude (Team 1-4).

At each altitude, the subjects were screened. Some were excluded when not suitable for testing. Exclusion criteria included cardiac illness, diuretic use, chronic medical conditions, or previous experience of AMS. All of the 88 qualified subjects were young males, of similar height (from 169 to $172 \mathrm{~cm}$ ) and weight (from 68 to $72 \mathrm{~kg}$ ), ranging in age from 20 to 24 years. They were divided into 5 groups, 34 at $3700 \mathrm{~m}$ (17 in each of Group 1 and 2), 18 at $4000 \mathrm{~m}$ (Group 3), 19 at $4700 \mathrm{~m}$ (Group 4), 17 at $5380 \mathrm{~m}$ (Group 5).

Additional 8 young men (22-24 years old) in Xi'an city were selected for the blood gas tests in hypobaric chamber.

The study was approved by the local institutional ethical review boards of all the participating institutions.

\section{Instrument}

To satisfy needs in high altitude, the OIR was invented (Figure 1, Chinese patent No. 200610104868.1, 200630090257.7, 200403262498.0). The total weight is $370 \mathrm{~g}$, and the dimensions are $152 \times 79 \times 34 \mathrm{~mm}$. As a simple, portable, durable, and convenient device, the OIR increases atmospheric pressure and therefore oxygen partial pressure in a nasal mask by means of a centrifugal fan with a rotational speed of $10000 \mathrm{rpm}$. 


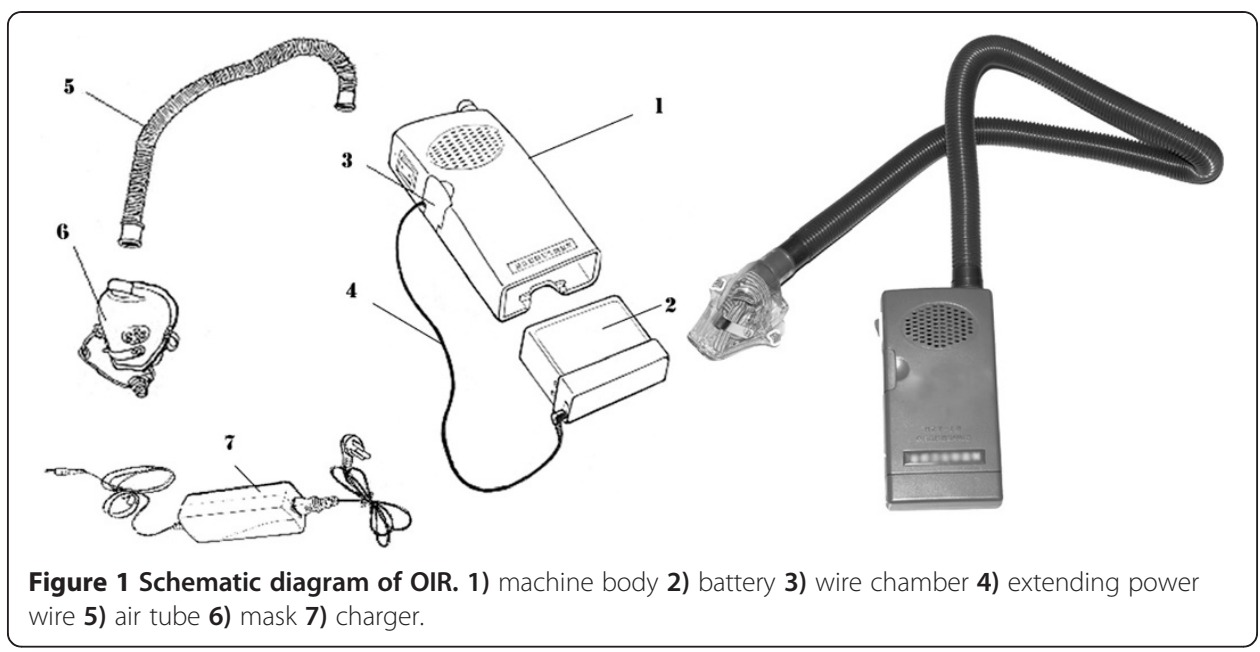

Magnetic suspension bearing was adopted to reduce noise and improve power consumption ratio. Conveniently, it uses internal rechargeable Li-ion batteries (11.1 V, 1800 $\mathrm{mAh}$ ) which, under extreme cold environment, can be displaced under the user's clothes with an extended wire connecting to the machine body to be kept warm and working.

The instrument does not change the percentage of oxygen (approximately 21\%) in the compressed atmosphere. There are 12 small holes $(2.5 \mathrm{~mm}$ in diameters $)$ in the mask allowing air exchanges. The output pressure in the mask, as measured using a U-tube manometer, is $3 \mathrm{mmHg}$ above the local atmospheric pressure; flow rate of atmosphere is approximately $50 \mathrm{~L} / \mathrm{min}$. The edge of the nasal mask is made of polysiloxanes, which can fit different faces and comfort the wearers.

\section{Experiment design}

The details of the experimental design are shown in Figure 2. The base at $3700 \mathrm{~m}$ has better facilities and two Groups (Group 1 and 2) were assigned with more tests performed than other groups. In Group 1, heart rate, $\mathrm{SpO}_{2}, \mathrm{MDA}, \mathrm{SOD}$ and BLA were tested. On day one, subjects wore the OIR. Team 1 recorded the subjects' heart rates and $\mathrm{SpO}_{2}$ at rest by a Multi-parameter patient monitor (IntelliVue MP70, Philips, Eindhoven, The Netherlands). Next, all subjects exercised using Harvard Step Test designed to induce fatigue [12]. The height of each step is $40 \mathrm{~cm}$; subjects went up and down at a rate of 20 steps per minute for 3 minutes. The heart rate and $\mathrm{SpO}_{2}$ were recorded at the end of exercise, and the recovery of heart rate was tracked during a rest period of 5 minutes. Immediately after the excise, the vein blood was drawn from the antecubital fossa to assess the concentrations of MDA [13], SOD [14], and BLA with three

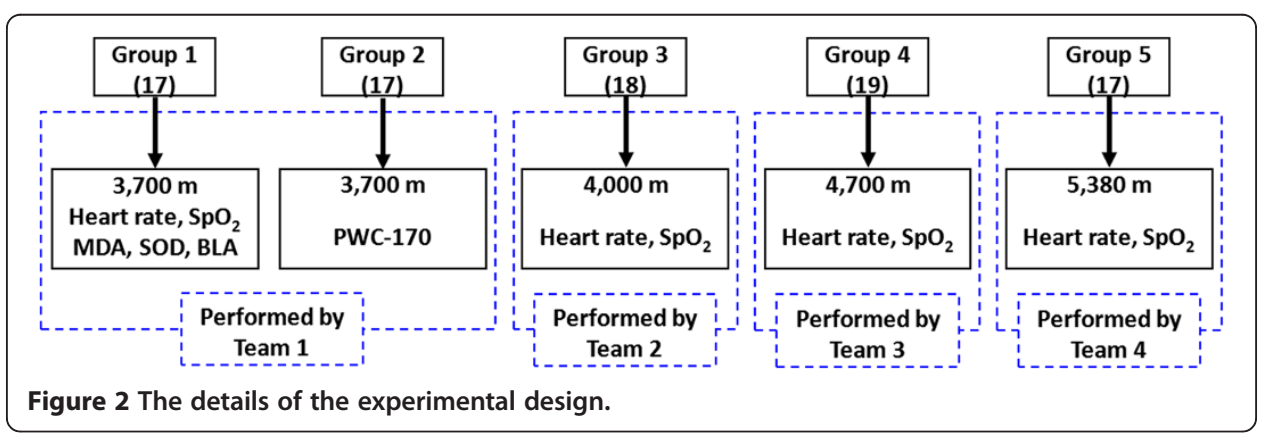


diagnostic kits (Jiancheng Bioengineering Institute, Nanjing, China) [15]. The experimental protocol was repeated without OIR on day two after a rest period of 24 hours.

PWC-170 [16] were tested from another 17 subjects in Group 2. They performed consecutive workloads on a cycle ergometer (EGM-II, Yueyang Electronic Ins., Co., Hunan, China) with OIR. Subjects were asked to keep the tachometer at around $60 \mathrm{rpm}$. Loaded power of the ergometer was initially set to $50 \mathrm{~W}$, then increased by a step of $50 \mathrm{~W}$ per 3 minutes until reaching $200 \mathrm{~W}$. Heart rate was monitored until a steady read-out was achieved. Each steady-state heart rate and workload were graphed, with the line of best fit for the three points extrapolated to estimate the power out that would elicit a heart rate of 170 beats per minute [16,17]. The experimental protocol was repeated without OIR after a rest of 24 hours.

At higher altitude (4,000 m, 4,700 m and 5,380 m for Group 3, 4, 5 respectively), only heart rate and $\mathrm{SpO}_{2}$ were measured from subjects exercising Harvard Step Test with the same type of patient monitor. Subjects performed the experiments first with OIR and then repeated without OIR after a rest of 24 hours.

An additional experiment was conducted to verify the exact arterial partial pressure of oxygen in blood $\left(\mathrm{PaO}_{2}\right)$ and arterial oxygen saturation $\left(\mathrm{SaO}_{2}\right)$ by measuring arterial blood gas with a portable clinical analyzer (i-STAT 200, Abbott Point of Care Inc., USA). Eight young men were exposed in the hypobaric chamber simulating high altitude of $4000 \mathrm{~m}$. Blood samples were collected from each subject after 2 hours exposure (without OIR). Afterwards, OIRs were used for 15 minutes and blood samples were collected (with OIR).

\section{Statistical analysis}

All of the data are shown as means \pm S.D. We used SPSS 13.0 software (SPSS Inc., Chicago, IL, USA) to perform Shapiro-Wilk normality test and paired $t$-test. $\mathrm{P}<0.05$ was considered statistically significant.

\section{Results}

Both in the situations of rest and of exercise, the results showed that the subjects with OIR had a higher $\mathrm{SpO}_{2}$ than the same subjects without OIR $(\mathrm{P}<0.01$ in exercise at $4700 \mathrm{~m}, \mathrm{P}<0.05$ in other circumstance, Figure 3). Compared with the 'without OIR'

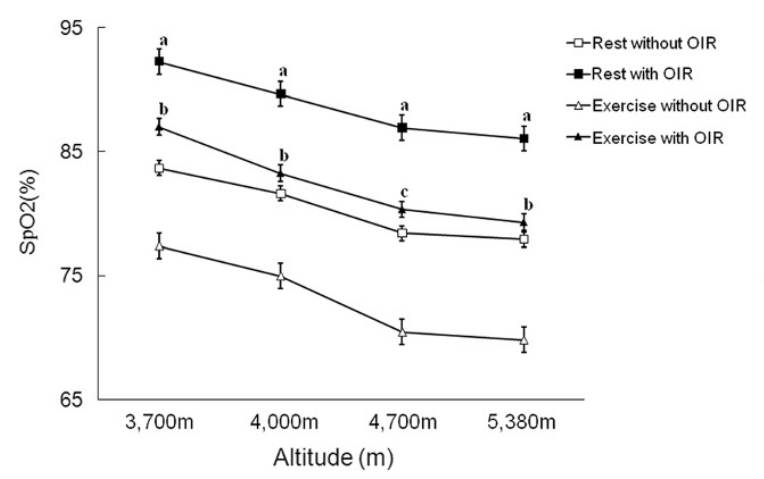

Figure 3 Effects of OIR on SaO2 at high altitudes of 3,700 m, 4,000 m, 4,700 m, and 5,380 m. $P$ values were estimated using paired Student's t-test. aP $<0.05$, as compared with 'Rest without OIR'. $\mathrm{bP}<0.05$ and $\mathrm{CP}<0.01$, as compared with 'Motion without OIR'. 


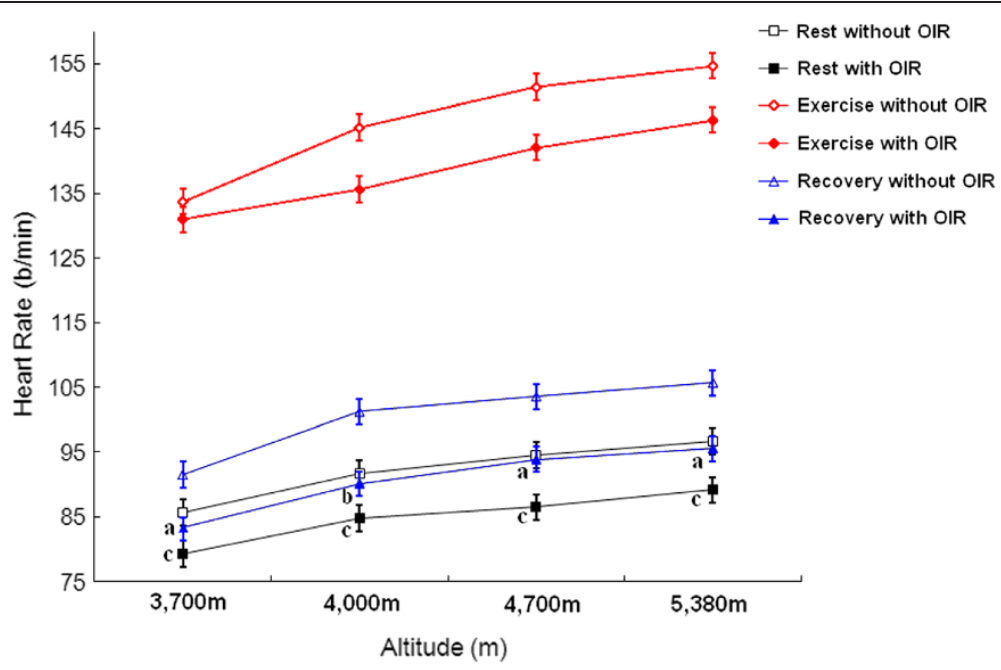

Figure 4 Effects of OIR on heart rate at high altitudes of 3,700 m, 4,000 m, 4,700 m, and 5,380 m. a $P<0.05$ and $b \mathrm{P}<0.01$, as compared with 'Recovery without OIR'. c $\mathrm{P}<0.05$, as compared with 'Rest without OIR'.

group, subjects with OIR had a lower heart rate at rest and better recovery of heart rate after exercise $(\mathrm{P}<0.01$ in recovery at $4000 \mathrm{~m}, \mathrm{P}<0.05$ in other circumstance, Figure 4$)$; however, there was no significant difference in heart rate during exercise between the two conditions. Compared with 'without OIR', subjects 'with OIR' in Group 1 had lower concentrations of MDA and BLA, and a higher concentration of SOD $(\mathrm{P}<0.05$, Table 1$)$. Based on the performances of PWC-170, subjects with OIR presented higher physical capacity. The power output of the subjects with OIR was better than that when without OIR $(\mathrm{P}<0.05$, Table 2$)$.

Comparing the results from Group 3, 4 and 5, we found the following: First, the $\mathrm{SpO}_{2}$ with OIR was higher than that without OIR both at rest and during exercise (Figure 3). Second, the heart rate with OIR was lower than that without OIR at rest and during the 5-minute recovery period (Figure 4).

In the simulation experiment, we found that $\mathrm{PaO}_{2}$ and $\mathrm{SaO}_{2}$ at altitude of $4000 \mathrm{~m}$ simulated in the hypobaric chamber were higher for the 'with OIR' group than for 'without OIR' group $(\mathrm{P}<0.05$, Table 3$)$.

\section{Discussion}

The OIR was developed with the aim of protecting unacclimatized people who must ascend to high altitude. The edge of the nasal mask is made of polysiloxanes, which can fit different faces and make wearers feel comfortable. In our study, we tested the effects of OIR at high plateau in subjects (young men aged 20-24 years old) at conditions of

Table 1 Changes in MDA, SOD, and BLA at 3,700 $\mathrm{m}($ Mean $\pm \mathrm{SD}, \mathrm{N}=17)$

\begin{tabular}{lccc}
\hline & MDA $(\boldsymbol{\mu m o l} / \mathbf{L})$ & BLA $(\mathbf{m m o l} / \mathbf{L})$ & SOD $(\mathbf{U} / \mathbf{L})$ \\
\hline Without OIR & $4.51 \pm 0.22$ & $6.37 \pm 0.53$ & $95.35 \pm 13.59$ \\
\hline With OIR & $4.33 \pm 0.25^{*}$ & $5.42 \pm 0.56^{*}$ & $112.62 \pm 12.93^{*}$
\end{tabular}

${ }^{*} \mathrm{P}<0.05$. 'Without OIR' vs. 'With OIR'. P values were estimated using paired Student's $t$-test. MDA: Malonodialdehyde, SOD: Superoxide dismutase, BLA: Blood lactate. 
Table 2 Increased output Power (watt) by using OIR in the PWC-170 test (Mean \pm SD, $\mathbf{N}=17)$

\begin{tabular}{llll}
\hline Heart rate range(BPM) & $\mathbf{1 1 5} \mathbf{- 1 3 0}$ & $\mathbf{1 3 0} \mathbf{- 1 4 5}$ & $\mathbf{1 7 0}^{\mathbf{e}}$ \\
\hline Without OIR & $100.9 \pm 12.1$ & $158.2 \pm 16.2$ & $196.3 \pm 15.5$ \\
\hline With OIR & $119.6 \pm 9.1^{*}$ & $182.1 \pm 10.5^{*}$ & $223.5 \pm 11.3^{*}$ \\
\hline $\begin{array}{l}\text { 170 } \\
\text { *P } \text { :These data were estimated by three consecutive workloads. }\end{array}$ & \\
\hline
\end{tabular}

rest or exercise. Subjects with OIR had lower heart rates (except during exercise), lower concentrations of MDA and BLA, higher levels of $\mathrm{SpO}_{2}$ and concentration of SOD, and better physical capacity than the same subjects without OIR. Some researchers reported similar findings in unsystematic and single-trial studies using smaller samples and/or different methods $[18,19]$. However, we have to point out that the subjects in this study are healthy male subjects and results may differ for other subjects.

The percentage of oxygen in air is constant, remaining at $21 \%$ in high altitude; however, atmospheric pressure is inversely related to altitude, making the amount of oxygen available much less in high altitude. The effect of ascending high altitude without acclimatization is a pathological condition that is caused by acute exposure to low atmospheric pressure [1]. Therefore, the key factor of AMS was the partial pressure of oxygen, the product of overall pressure and fractional concentration of oxygen.

The oxyhemoglobin dissociation curve describes the non-linear relation between the partial pressure of oxygen in the blood and the oxygen saturation. The sigmoid shape of the curve indicates that small increase in partial pressure of oxygen can lead to high increase of $\mathrm{SaO}_{2}$. In our study, although the OIR just slightly increases the oxygen partial pressure of inspiratory gas in the airway (the output pressure of OIR is measured at about $3 \mathrm{mmHg}$ above the local atmospheric pressure), it significantly increases the $\mathrm{SpO}_{2}$ (about 12.8\%). Caution must be taken that the added partial pressure of oxygen by using the OIR is exerted in the airway and not necessarily equally transferred into the blood. In fact, we did an additional experiment verifying the exact arterial partial pressure of oxygen in blood by measuring arterial blood gas. The additional experiment on blood gas confirmed the $\mathrm{SaO}_{2}$ in blood is high with OIR than without OIR.

$\mathrm{SpO}_{2}$ is the indirect measure (by a pulse oximetry at fingertip) of the ratio of oxyhemoglobin to the total concentration of hemoglobin present in the blood. Heart rate has a close relationship to oxygen consumption, and heart rate increases following ascent to high altitude. The recovery of heart rate, measured at a fixed (or reference) period after ceasing activity, is correlated to some factors which affect cardiovascular functions [20]. In 'with OIR' condition, OIR significantly enhanced $\mathrm{SpO}_{2}$ and heart rate recovery after exercise and reduced heart rate during rest at all altitudes. In particular, $\mathrm{SpO}_{2}$ at $4,700 \mathrm{~m}$ and heart rate recovery at $4,000 \mathrm{~m}$ were highly significantly different between with and without OIR. A higher level of $\mathrm{SpO}_{2}$ should protect cardiopulmonary

Table 3 Arterial blood gas and oxygen saturation in altitude of $\mathbf{4 0 0 0}$ m simulated in the hypobaric chamber (Mean \pm SD, $n=8$ )

\begin{tabular}{llccc}
\hline & $\mathbf{p H}$ & $\mathbf{P a C O}_{\mathbf{2}} \mathbf{( \mathbf { m m H g } )}$ & $\mathbf{P a O}_{\mathbf{2}}(\mathbf{m m H g})$ & $\mathbf{S a O}_{\mathbf{2}} \mathbf{( \% )}$ \\
\hline Without OIR & $7.45 \pm 0.03$ & $35.2 \pm 2.9$ & $55.0 \pm 3.5$ & $68.3 \pm 5.9$ \\
\hline With OIR & $7.49 \pm 0.05$ & $31.3 \pm 4.2$ & $63.2 \pm 3.3^{*}$ & $80.3 \pm 9.4^{*}$ \\
\hline
\end{tabular}

$\mathrm{PaCO}_{2}$ : the partial pressure of carbon dioxide; $\mathrm{PaO}_{2}$ : the partial pressure of oxygen; $\mathrm{SaO}_{2}$ : arterial oxygen saturation. ${ }^{*} \mathrm{P}<0.05$. 'Without OIR' vs. 'With OIR'. P values were estimated using paired Student's $t$-test. 
function during acute exposure to low atmospheric pressure, and may also play an important role in reducing heart overload.

Lassitude is one of the symptoms of altitude sickness [21]. Erythrocyte membranes are perhaps the most exposed to peroxidative damage by free radicals [22]. In low atmospheric pressure conditions, the imbalance between oxidation and antioxidation reactions results in a higher concentration of MDA, which further impairs cell function [23] and damages cellular membrane components [24]. BLA is used clinically as an indicator of circulatory impairment as well as the overall state of oxygenation [25]. Antioxidant defenses in the red cell can temper the negative effect of free radicals and related reactions and keep them in check. SOD forms a substantial defense network against oxidative stress imposed by physical activity [3]). Subjects in Group 1 with OIR had lower concentrations of MDA and BLA, and a higher concentration of SOD $(\mathrm{P}<0.05)$ at $3,700 \mathrm{~m}$, compared with when without OIR. This suggests that OIR works by reducing lipid peroxidation and cell membrane damage, thereby increase the body's ability to fight anoxia and fatigue.

Flueck et al. [11] reported that the physical capacity for work can be tested in young males aged $17-24$ by means of the PWC-170 test. The PWC-170 has also been shown to be a valid tool at high altitude [26]. The OIR increased air flow capacity by the rotating fan and accumulated the volume of oxygen. The higher total air pressure and oxygen saturation in subjects using OIR sustained normal physical alertness in high altitude, as assessed by their performance in the PWC-170 test.

OIR has two known limitations. First, OIR has not been proved to treat AMS, despite the data showing better performance. Second, OIR may cause irritation of the respiratory tract when air temperature is low. One limitation of the experimental design is that all the subjects performed experiments with OIR first. In future work, an independent experiment concerning the sequence of with and without OIR should be designed to reveal its effects on the results.

\section{Conclusions}

The OIR not only increased the level of $\mathrm{SpO}_{2}$, concentration of SOD, and physical capacity but also reduced the heart rate (except during exercise) and concentrations of MDA and BLA, which play important protective roles at low atmospheric pressures and reduce the risk of AMS. We suggested that OIR may play a useful role in protecting people needing to ascend to high altitude before acclimatization. 


\section{References}

1. Hackett P, Roach R: Current concepts high-altitude illness. N Engl J Med 2001, 345:107-114.

2. Kleinsasser $A$, Loeckinger $A$ : Brief report: pressure support ventilation during an ascent and on the summit of Mt. Everest? A theoretical approach. High Altitude Medicine \& Biology 2002, 1:65-68.

3. Bartsch P, Merki B, Hofstetter D, Maggiorini M, Kayser B, Oelz O: Treatment of acute mountain sickness by simulated descent: a randomised controlled trial. British Medical Journal 1993, 6885:1098-1101.

4. Imray C, Clarke T, Forster P, Harvey T, Hoar H, Walsh S, Wright A: Carbon dioxide contributes to the beneficial effect of pressurization in a portable hyperbaric chamber at high altitude. Clin Sci 2001, 2:151-157.

5. Stream J, Grissom C: Update on high-altitude pulmonary edema: pathogenesis, prevention, and treatment. Wilderness and Environmental Medicine 2008, 4:293-303.

6. West J: Commuting to high altitude: value of oxygen enrichment of room air. High Altitude Medicine \& Biology 2002, 2:223-235.

7. Markovic D, Kovacevic H: Recompression therapy of mountain sickness. Arhiv Za Higijenu Rada i Toksikologiju 2002, 1:3-6.

8. West J: Oxygen enrichment of room air to relieve the hypoxia of high altitude. Respir Physiol 1995, 2:225-232.

9. Rodway G, Windsor J, Hart N: Supplemental oxygen and hyperbaric treatment at high altitude: cardiac and respiratory response. Aviation Space \& Environmental Medicine 2007, 6:613-617.

10. Bailey D, Evans K, James P, McEneny J, Young I, Fall L, Gutowski M, Kewley E, McCord J, Møller K: Altered free radical metabolism in acute mountain sickness: implications for dynamic cerebral autoregulation and bloodbrain barrier function. J Physiol 2009, 1:73-85.

11. Flueck M: Plasticity of the muscle proteome to exercise at altitude. High Altitude Medicine \& Biology 2009, 2:183-193.

12. Francis K: Fitness assessment using step tests. Compr Ther 1987, 4:36-41.

13. Huang H, Han C, Yan H, Kao W, Tsai C, Yen D, Huang C, Chen W: Oxidative stress and erythropoietin response in altitude exposure. Clinical \& Investigative Medicine 2008, 6:E380-E385.

14. Wozniak A, Drewa G, Chesy G, Rakowski A, Rozwodowska M, Olszewska D: Effect of altitude training on the peroxidation and antioxidant enzymes in sportsmen. Medicine and Science in Sports and Exercise 2001, 7:1109-1113.

15. Cerretelli P, Samaja M: Acid-base balance at exercise in normoxia and in chronic hypoxia. Revisiting the" lactate paradox". Eur J Appl Physiol 2003, 5:431-448.

16. Svannshvili R, Sopromadze Z, Kakhabrishvili Z, Svannshvili T, Maskhuliia L: Athletes' physical working capacity. Georgian Medical News 2009, 166:68-73.

17. Renner F, Haber P, Rainer P: Results of electrocardiographic examinations and exercise tests in 18 year old male recruits. Zeitschrift fur Kardiologie 1981, 6:495-499.

18. Cui J, Xu L, Bressler S, Ding M, Liang H: BSMART: A Matlab/C toolbox for analysis of multichannel neural time series. Neural Networks 2008, 8:1094-1104.

19. Luo E, Shen G, Wu X, Lu L, Xu Q: Effect of oxygen-increased respirator on $\mathrm{SaO} 2$ and heart rate under plateau environment. Space Medicine \& Medical Engineering 2005, 4:297-299.

20. Morshedi-Meibodi A, Larson MG, Levy D, O'Donnell CJ, Vasan RS: Heart rate recovery after treadmill exercise testing and risk of cardiovascular disease events (The Framingham Heart Study). Am J Cardiol 2002, 8:848-852

21. Gudmundsson G, Gudbjartsson T: High altitude sickness-review. Læknabla i 2009, 6:441-447.

22. Bailey D, Evans K, James P, McEneny J, Young I, Fall L, Gutowski M, Kewley E, McCord J, Møller K: Altered free radical metabolism in acute mountain sickness: implications for dynamic cerebral autoregulation and bloodbrain barrier function. J Physiol (Lond.) 2009, 1:73-85.

23. Courtois F, Seidman E, Delvin E, Asselin C, Bernotti S, Ledoux M, Levy E: Membrane peroxidation by lipopolysaccharide and iron-ascorbate adversely affects Caco-2 cell function: beneficial role of butyric acid. Am J Clin Nutr 2003, 3:744-750.

24. Karar J, Dolt K, Mishra M, Arif E, Javed S, Pasha M: Expression and functional activity of pro-oxidants and antioxidants in murine heart exposed to acute hypobaric hypoxia. FEBS Lett 2007, 24:4577-4582.

25. Toffaletti J: Elevations in blood lactate: overview of use in critical care. Scand I Clin Lab Investig 1996, S224:107-110.

26. Bircher $\mathrm{H}$, Eichenberger $\mathrm{U}$, Maggiorini $\mathrm{M}$, Oelz $\mathrm{O}$, Bärtsch P: Relationship of mountain sickness to physical fitness and exercise intensity during ascent. Journal of Wilderness Medicine 1994, 3:302-311.

doi:10.1186/1475-925X-11-49

Cite this article as: Shen et al:: The role of oxygen-increased respirator in humans ascending to high altitude. BioMedical Engineering OnLine 2012 11:49. 